\title{
EFEITO DO PROBIÓTICO APÓS TOXICIDADE HEPÁTICA DO DICROMATO DE POTÁSSIO EM RATOS
}

Reinaldo Camacho Bezerra ${ }^{1}$; Paulo Eduardo Pardo ${ }^{2}$; Gisele Alborghetti Nai ${ }^{1}$; Hermann BremerNeto $^{1}$; Rogério Giuffrida ${ }^{1}$

${ }^{1}$ Universidade do Oeste Paulista - UNOESTE. Mestrado em Ciência Animal, Presidente Prudente - SP. ${ }^{2}$ Médico Veterinário, Presidente Prudente - SP. Email: camacho reinaldo@hotmail.com.

\section{RESUMO}

O objetivo do presente trabalho foi o de avaliar as alterações dependentes da dose de dicromato de potássio $\left(0,12,24\right.$ e $\left.36 \mathrm{mg} \cdot \mathrm{kg}^{-1}\right)$ no tecido hepático, após suplementação com probiótico em dosagens 0 ou $0,2 \%$, em 80 ratos machos. A ingestão oral por 90 dias de doses crescentes de dicromato de potássio produziu sinais clínicos de toxicidade frente à análise histopatológica $(p<0,05)$ e atividades séricas enzimáticas $(p<0,05)$, dos marcadores de função hepática. A inclusão do probiótico na dieta mitigou os efeitos nos parâmetros estudados.

Palavras-chave: crômio vi; alimentos funcionais; atividade sérica enzimática; histopatologia; ratos.

\section{EFFECT OF PROBIOTIC AFTER HEPATIC TOXICITY OF POTASSIUM DICHROMATE IN RATS}

\begin{abstract}
ABSTRAT
This work aims to evaluate the alterations, depending on the dose of potassium dichromate $(0,12$, 24 and $\left.36 \mathrm{mg} \cdot \mathrm{kg}^{-}{ }^{1}\right)$, in liver tissue after supplementation with probiotic at dosages 0 or $0.2 \%$ in 80 male rats. Oral ingestion done for 90 days of increasing amounts of potassium dichromate produced clinical signs of toxicity compared to histopathological analysis $(p<0.05)$, and seric enzymatic activities $(p<0.05)$, markers of hepatic function. The inclusion of probiotics to the diet mitigated the effects on the studied parameters.
\end{abstract}

Keywords: chromium vi; intoxication; seric enzymatic activity; histopathology; rats. 


\section{INTRODUÇÃO}

O crômio ( $\mathrm{Cr}$ ) é um elemento que ocorre naturalmente, e em abundancia na crosta terrestre (WITT et al., 2013), sendo um dos elementos essenciais que desempenham papel no metabolismo de glicose e lipídeos, podendo existir em vários estados de valência. $\mathrm{Na}$ natureza, $\mathrm{o} \quad \mathrm{Cr}^{+3} \mathrm{em}$ pequenas quantidades é considerado um elemento essencial para a vida humana (NEILL et al., 2012). Já o $\mathrm{Cr}^{+6}$, segundo a U. S. EPA (2003), não ocorre naturalmente, sendo considerado um contaminante, proveniente de fontes antropogênicas.

$\mathrm{O}$ dicromato de potássio $\left(\mathrm{K}_{2} \mathrm{Cr}_{2} \mathrm{O}_{7}\right)$ é a forma mais tóxicas do crômio (VI) (FUJIKAWA, 2002; SEDMAN et al., 2006).

Alguns microorganismos vivos, definidos como probióticos, mostraram atividade antioxidante (SINGH et al., 2007) e captadora de radicais livres (GETOFF, 2007), o que pode minimizar e/ou impedir o efeito tóxico de contaminantes. As bactérias do ácido lático são as principais representantes dos probióticos em alimentos e produtos farmacêuticos e dentre essas podemos incluir muitas espécies de Lactobacillus, Bifidobacterium, Streptococcus e ainda alguma cepas não patogênicas da Escherichia coli (PARDO; REIS, 2008; ALMEIDA et al., 2013).

O objetivo desse estudo foi avaliar o efeito de proteção hepática do probiótico em ratos Wistars, alimentados com rações contendo doses crescentes de dicromato de potássio.

\section{METODOLOGIA}

O presente estudo experimental foi aprovado pela Comissão de Ética em Uso de Animais da Universidade do Oeste Paulista, segundo protocolo de aprovação no784/2012.

Foram utilizados 80 ratos, machos jovens. Sendo mantidos em gaiolas individuais, sob as mesmas condições de padrão de iluminação. Onde o experimento teve duração de 97 dias, sendo 7 dias para adaptação ao manejo. 
Tabela 1. Representação das dietas ofertadas aos diferentes grupos durante o experimento.

\begin{tabular}{|c|c|c|c|c|}
\hline Grupos & № de animais & Crômio & Probiótico & Característica \\
\hline G1 & 10 & Ausente & Ausente & Controle \\
\hline G2 & 10 & $\begin{array}{c}12 \mathrm{mg} \cdot \mathrm{kg}^{-1} \\
\mathrm{~K}_{2} \mathrm{Cr}_{2} \mathrm{O}_{7}\end{array}$ & Ausente & Agressão \\
\hline G3 & 10 & $\begin{array}{c}24 \mathrm{mg} \cdot \mathrm{kg}^{-1} \\
\mathrm{~K}_{2} \mathrm{Cr}_{2} \mathrm{O}_{7}\end{array}$ & Ausente & Agressão \\
\hline G4 & 10 & $\begin{array}{c}36 \mathrm{mg} \cdot \mathrm{kg}^{-1} \\
\mathrm{~K}_{2} \mathrm{Cr}_{2} \mathrm{O}_{7}\end{array}$ & Ausente & Agressão \\
\hline G5 & 10 & Ausente & $\begin{array}{c}0,2 \% \\
\text { Probiótico }\end{array}$ & Controle \\
\hline G6 & 10 & $\begin{array}{c}12 \mathrm{mg} \cdot \mathrm{kg}^{-1} \\
\mathrm{~K}_{2} \mathrm{Cr}_{2} \mathrm{O}_{7}\end{array}$ & $\begin{array}{c}0,2 \% \\
\text { Probiótico }\end{array}$ & Agressão/Tratamento \\
\hline G7 & 10 & $\begin{array}{c}24 \mathrm{mg} \cdot \mathrm{kg}^{-1} \\
\mathrm{~K}_{2} \mathrm{Cr}_{2} \mathrm{O}_{7}\end{array}$ & $\begin{array}{c}0,2 \% \\
\text { Probiótico }\end{array}$ & Agressão/Tratamento \\
\hline G8 & 10 & $\begin{array}{c}36 \mathrm{mg} \cdot \mathrm{kg}^{-1} \\
\mathrm{~K}_{2} \mathrm{Cr}_{2} \mathrm{O}_{7}\end{array}$ & $\begin{array}{c}0,2 \% \\
\text { Probiótico }\end{array}$ & Agressão/Tratamento \\
\hline
\end{tabular}

As dietas sólidas e hídricas foram fornecidas ad libitum. Após o período experimental, os animais foram anestesiados com Tiopental (Produtos Químicos Farmacêuticos Ltda. - São Paulo/SP - Brasil), e amostras de sangue de todos os ratos foram colhidas por punção intracardíaca. Foram determinados os seguintes marcadores de função hepática: Aspartato Aminotransferase (AST), Alanina Aminotransferase $(A L T)$, Fosfatase Alcalina (ALP) e Gama Glutamiltransferase (GGT). Os parâmetros bioquímicos foram dosados através do analisador automatizado bioPLUS, modelo Bio200F. Após coleta das amostras de sangue, os animais foram eutanasiados, por injeção de $2 \mathrm{mg} / \mathrm{kg}$ de Tiopental (Produtos Químicos Farmacêutico Ltda. - São Paulo/SP - Brasil). Onde foi colhido o fígado para análise histopatológica. A mesma foi realizada de forma cega por um avaliador (GAN) utilizando-se microscópio óptico comum (NIKON Labophot, Japão) e avaliou-se os seguintes parâmetros, com seus respectivos escores: Congestão Tecidual, Intensidade do Infiltrado Inflamatório, Necrose, Colestase; Esteatose Microvesicular 
e Macrovesicular, Células de Kupffer e Neoplasia.

As comparações estatísticas para a análise histopatológica foram realizadas pelo teste não paramétrico de Kruskall-Wallis, seguido dos contrastes de Dunn para comparação entre grupos adotando significância de $p<0,05$. Já as comparações estatísticas das atividades séricas enzimáticas foram realizadas pelo teste não paramétrico de Levene seguido de ANOVA com contrastes de Tukey ou Games-Howell adotando significância de $p<0,05$.

\section{RESULTADOS}

Os resultados das análises das atividades séricas enzimáticas de função hepática (AST, ALT, FA e GGT) estão apresentados nas Figuras 1 a 4, enquanto a análise histopatológica tem sua representação expressa na Figura 5.

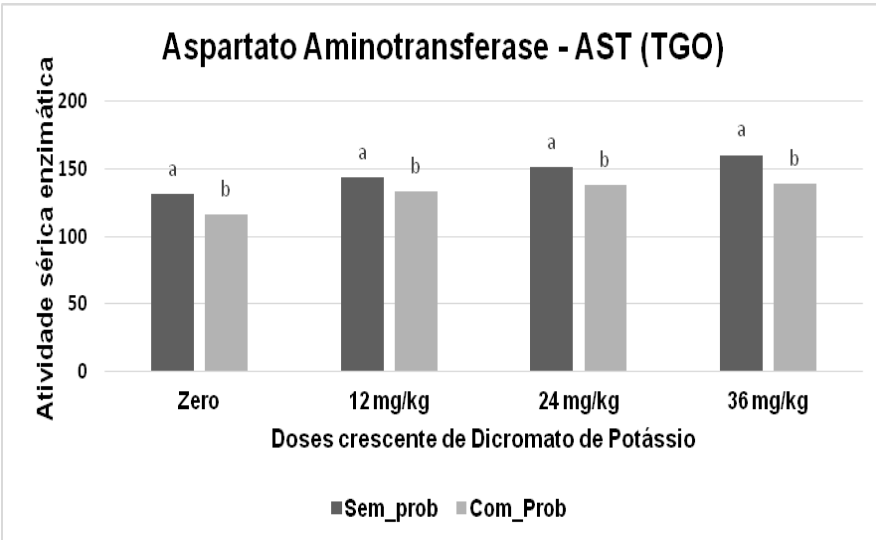

Figura 1. Atividade sérica AST nas diferentes doses. Diferentes sobrescritos diferem significativamente.

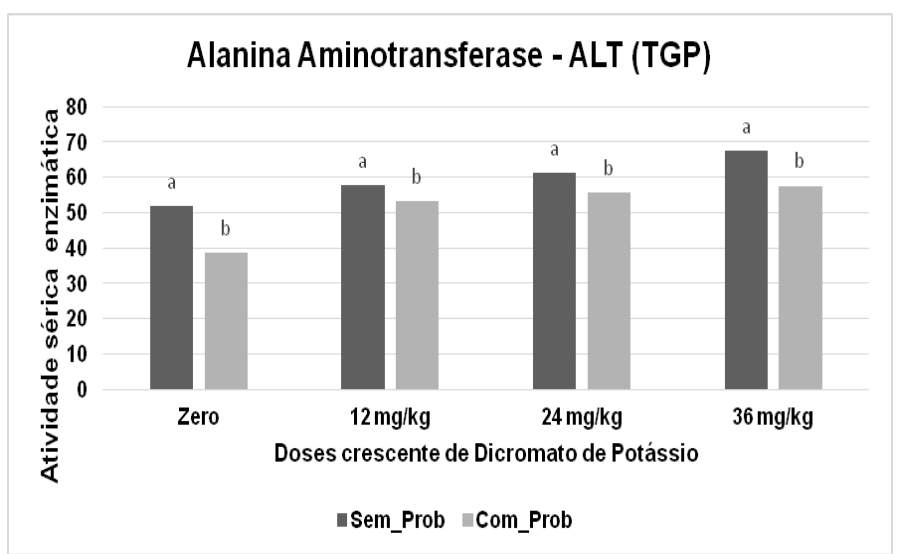

Figura 2. Atividade sérica ALT nas diferentes doses. Diferentes sobrescritos diferem significativamente. 


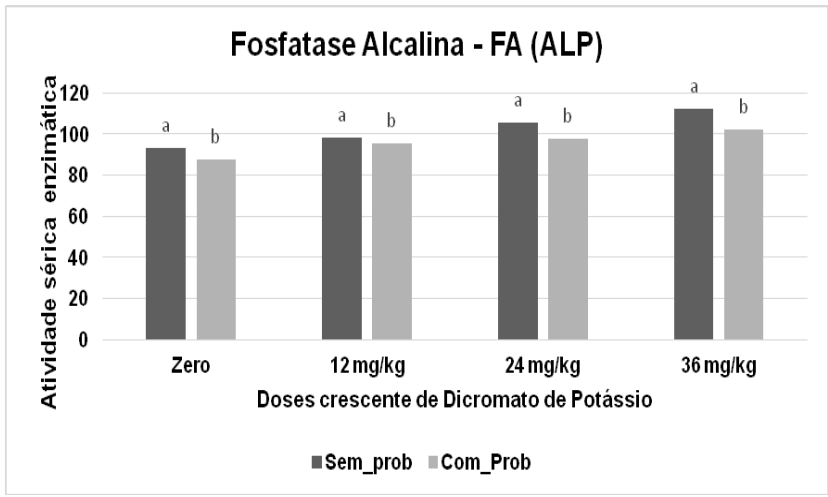

Figura 3. Atividade sérica ALP nas diferentes doses. Diferentes sobrescritos diferem significativamente.

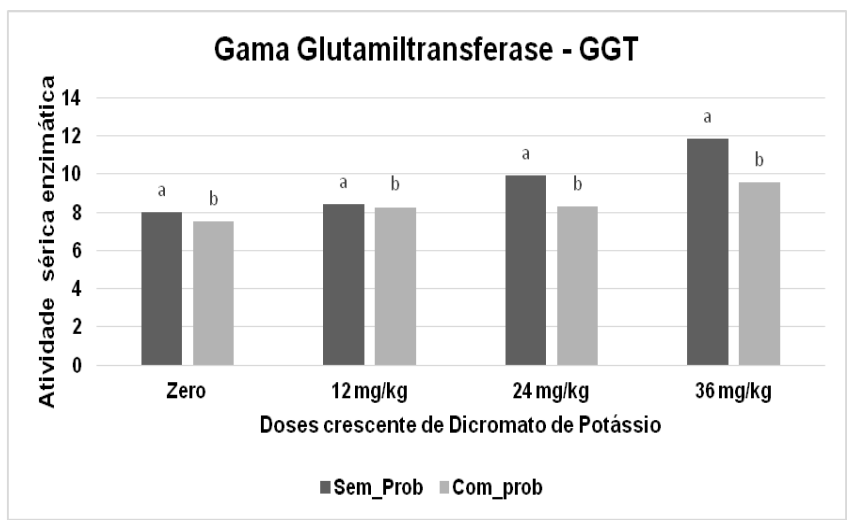

Figura 4. Atividade sérica GGT nas diferentes dietas. Diferentes sobrescritos diferem significativamente.
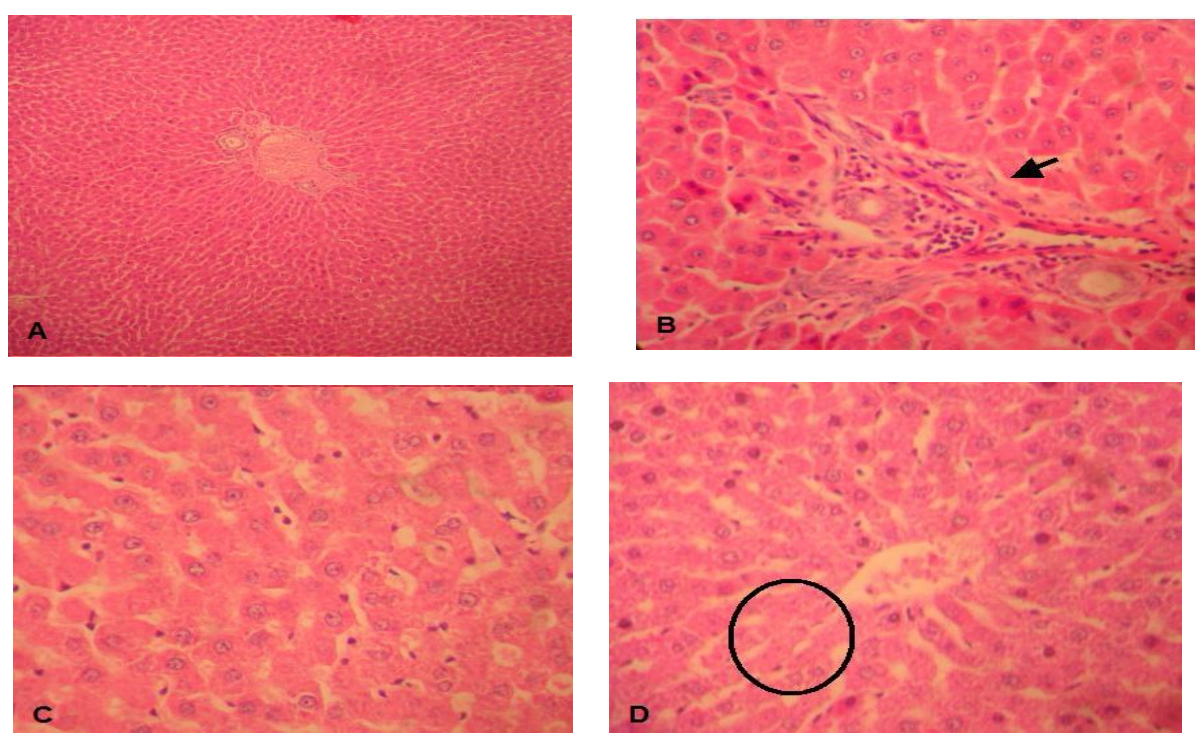

Figura 5. Fotomicroscopia do fígado. A - Fígado normal, sem aumento de células de Küpffer animal do grupo G1 (Hematoxilina-eosina, aumento de 100x). B - Foco inflamatório (seta) e ausência de aumento de células de Küpffer - animal do grupo G2 (Hematoxilina-eosina, aumento de 400x). C - Esteatose microvesicular difusa e aumento de células de Küpffer - animal do grupo G3 (Hematoxilina-eosina, aumento de 400x). D - Foco de necrose hepatocítica (círculo), esteatose microvesicular e ausência de aumento de células de Küpffer - animal do grupo G4 (Hematoxilinaeosina, aumento de 400x). 
DISCUSSÃO

Durante 90 dias de experimento não houve nenhum caso de morte entre os animais.

Dois animais do grupo (G3) na qual foi adicionado 24 mg. $\mathrm{kg}^{-1}$ de dicromato de potássio, sem adição de probiótico e quatro no grupo (G4), na qual foi adicionado 36 $\mathrm{mg} . \mathrm{kg}^{-1}$ apresentaram sintomas de apatia, letargia e diarreia, corroborando com os relatos em ratos por Priti et al. (2012), em seres humanos por Zhang e Li (1987) e em coelhos por Tyl et al. (1991), após a exposição ao $\mathrm{Cr}^{+6}$. Segundo Saryan e Reedy (1988) e Priti et al. (2012) a possível razão para esses sintomas pode ser devido aos efeitos corrosivos e irritantes do sal hexavalente na mucosa gastrointestinal.

Os grupos que receberam doses crescentes de $\mathrm{Cr}^{+6}$ incorporado a $0,2 \%$ de probiótico Proenzime ${ }^{\oplus}$ não apresentaram sintomas de embotamento, apatia, letargia e ou diarreia, indicando que esse produto diminuiu a absorção do $\mathrm{Cr} \quad\left(\mathrm{K}_{2} \mathrm{Cr}_{2} \mathrm{O}_{7}\right)$ e protegeu a mucosa gastrointestinal dos efeitos corrosivos e irritantes do crômio.

Os resultados das análises das atividades séricas enzimáticas de função hepática (AST, ALT, FA e GGT) estão apresentados nas Figuras 1 a 4, enquanto a análise histopatológica tem sua representação expressa na Figura 5.
Segundo Brochers et al. (2009), o papel das bactérias probióticas na melhora da imunidade, pode ser explicada por meio da atividade de desintoxicação desses microorganismos, pois agora existe evidências substanciais de que podem proporcionar benefícios modulando as funções imunológica, antipatogênica e antiinflamatória (ADOLFSSON et al., 2004), possivelmente influenciando as funções metabólica, imunológica e protetora do cólon (ROBERFROID et al., 1995).

No presente estudo, quando os animais receberam dietas com ração incorporada a doses crescentes de dicromato de potássio, ocorreu um aumento significativo $(p<0,05)$ nos valores de AST, ALT, ALP e GGT conforme as figuras de 1 a 4 em relação ao grupo controle, que recebeu somente probiótico. Esses resultados reportam os observados em ratos por Acharya et al. (2001) para AST e ALT, porém esse estudo não obteve os mesmos resultados quanto ao parâmetro GGT, possivelmente pelo menor tempo de experimento.

Funções do coração e fígado foram também avaliados por Islam et al. (2004) e Mustari e Ahmad (2011) e obtiveram resultados semelhantes ao nossos em relação aos parâmetros AST e ALT, em ratos suplementados com probiótico. Segundo eles - parâmetro bioquímico AST está 
amplamente distribuído em vários órgãos, tais como o coração e músculos esqueléticos, bem como no fígado, enquanto o ALT existe principalmente no fígado. A ALT e AST são geralmente utilizados como marcadores de dano hepático (RAZA et al., 2002; HAYES, 2007).

Na análise histopatológica, em relação ao infiltrado inflamatório, não foram observadas diferenças significativas entre os grupos ( $p>0,05)$ (Figura 5B), assim como para necrose hepatocelular ( $p>0,05)$ (Figuras 5D). Estes resultados não corroboram com 0 estudo de Soudani (2010), que obteve resultados significativos $(p<0,05)$ para esses parâmetros, fato possivelmente explicado pela maior dosagem de dicromato de potássio utilizada em seu trabalho.

Com relação a congestão tecidual, houve um aumento significativo $(p<0,05)$ para os grupos que tiveram dieta acrescida de dicromato de potássio em doses crescentes $\left(12,24\right.$ e $\left.36 \mathrm{mg} \cdot \mathrm{kg}^{-1}\right)$ fato que corrobora o estudo de Balakrishnan (2013). Porém, quando ocorreu a adição de $0,2 \%$ de probiótico na dieta acrescida de doses crescentes de dicromato de potássio $(12,24$ e $36 \mathrm{mg} \cdot \mathrm{kg}^{-1}$ ) os resultados não foram compatíveis, existindo um aumento significativo no grau de congestão de leve para moderada, fato possivelmente explicado por uma maior atividade de biotransformação hepática.
Em relação à esteatose hepática os resultados foram significativos $(p<0,05)$, existindo um aumento no grau de esteatose dos grupos com dietas crescentes de dicromato de potássio $\left(12,24\right.$ e $\left.36 \mathrm{mg} \cdot \mathrm{kg}^{-1}\right) \mathrm{e}$ para os grupos com dieta crescente de dicromato de potássio acrescida de $0,2 \%$ de probióticos (Figuras $5 \mathrm{C}$ e 5D). Esses resultados não concordam com o estudo de Xu et al. (2012), pois em seu estudo a utilização do probiótico diminui significativamente $\mathrm{o}$ grau de esteatose hepática, fato que pode ser explicado pela forma de indução a esteatose hepática, que no estudo de Xu et al. (2012) foi através de dieta rica em lipídeos. No parâmetro células de Kupffer também foram obtidos resultados significativos $(p<0,05)$ (Figura $5 C)$, sendo observadas diferenças entre os grupos com doses crescentes de dicromato de potássio (12, 24 e $\left.36 \mathrm{mg} \cdot \mathrm{kg}^{-1}\right)$ e doses crescentes de dicromato de potássio acrescido de $0,2 \%$ de probiótico, sugerindo um aumento as resposta imune do organismo frente as agressões realizadas pelo dicromato de potássio assim como a estimulação produzida pelos probióticos. Estes resultados corroboram o estudo de Soriano (2009), que demonstrou que a utilização de probióticos realiza a modulação da resposta imune do organismo mediante às alterações na flora intestinal do hospedeiro, aumentando assim 
a capacidade de resposta do organismo frente as agressões.

Para o parâmetro colestase também não houve aumento significativo $(p>0,05)$ entre os grupos estudados, mesmo existindo aumento significativo $(p<0,05)$ da atividade sérica enzimática para GGT, fato possivelmente explicado pelo período de 90 dias de exposição ao dicromato de potássio realizado em nosso estudo.

Nenhum dos animais expostos ao dicromato de potássio apresentaram neoplasia, fato que não corrobora com os estudos da The Internacional Programme On Chemical Safety (1976), que relata em seus estudos que doses acima de $10 \mathrm{mg} \cdot \mathrm{kg}^{-1}$ produz efeitos carcinogênicos para animais experimentais, e os efeitos crônicos da exposição ao crômio ocorrem no fígado e nos rins. Fato possivelmente explicado pelo tempo de exposição ao crômio realizado em nosso estudo.

\section{CONCLUSÃO}

A inclusão do probiótico na dieta mitigou os efeitos tóxicos no fígado, evidenciados atráves dos resultados bioquímicos séricos da função hepatica e histopatologicos, indicando que ele pode ser utilizado para desintoxicar e/ou impedir a ação desse mineral, porém, outros estudos devem ser realizados.

\section{REFERÊNCIAS}

ACHARYA, S.; MEHTA, K.; KRISHNAN, S.; et al. A subtoxic interactive toxicity study of ethanol and chromium in male Wistar rats. Alcohol, v.23, p.99-108, 2001.

http://dx.doi.org/10.1016/S07418329(00)00139-7

ADOLFSSON, O.; MEYDANI, S.N.; RUSSELL, R.M. Yogurt and gut function. Am. J. Clin. Nutr., v.80, p.245-256, 2004.

ALMEIDA, L.E.; GENARO, S.C.; GEROTI, T.C.; FRAZATTI-GALINA, N.M.; BATISTA, M.P.; GIUFFRIDA, R.; PARDO, P.E.; SOUZA, L.G. Utilização de probiótico sobre o ganho de peso em bovinos da raça nelore. Colloquium Agrariae, v.9, n.1. 2013.

BALAKRISHNAN, R. et al. Antioxidant activity of coated probiotic lactobacillus casei on chromium (vi) induced oxidative stress in rats. In: THE NATIONAL ACADEMY OF SCIENCES, INDIA SECTION B: BIOLOGICAL SCIENCES. Proceedings... p. 1-6, 2013.

BORCHERS, T.A.; CARLO, S.; FREDERICK J.M.; CARL, L.K.; GERSHWIN, M.E. Probiotics and Immunity. Journal of Gastroenterology, v.44, n.1, p.26-46, 2009. http://dx.doi.org/10.1007/s00535-008-2296-0

FUJIKAWA, E.S. Incorporação do resíduo "serragem cromada" em materiais de construção civil. 2002. 80 f. Dissertação (Mestrado em Engenharia Mecânica) Faculdade de Engenharia e Tecnologia, Universidade Estadual Paulista, Bauru, 2002. Disponível em: <http://www.teses.usp.br>. Acesso em: 15 jul. 2013.

GETOFF, N. Anti-aging and aging factors in life: the role of free radicals. Radiat. Phys. Chem., v.76, p. 1577-1586, 2007. http://dx.doi.org/10.1016/j.radphyschem.2007. $\underline{01.002}$ 
HAYES, W. Principles and methods of toxicology. 5. ed. New York: Informa Health Care, 2007. http://dx.doi.org/10.1201/b14258

ISLAM, M.W.; RAHMAN, M.M.; KABIR, S.M.L.; KAMRUZZAMAN, S.M.; ISLAM, M.M. Effect of probiotics supplementation on growth performance and certain haemato-biochemical parameters in broiler chickens. Bangladesh Journal of Veterinary Medicine. v.2, p.39-43, 2004.

MUSTARI, A.; AHMAD. N. Effects of probiotics on serum biochemical parameters in rats. Bangl. Vet., v.28, p.70-74, 2011.

NEILL, L. et al. State of the science of hexavalent chromium in drinking water. Water Research Foundation, 2012.

NTP. National Toxicology Program. Technical report on the toxicity studies of sodium dichromate dehydrate (CAS № 7789-12-0) administered in drinking water to male and female $\mathrm{F} 344 / \mathrm{N}$ rats and B6C3F1 mice and male $\mathrm{BALB} / \mathrm{c}$ and am 3-C57BL/6 mice. Washington: National Toxicology Program, 2007. Toxicity Report Series Number 72. Available: <http://ntp.niehs.nih.gov/ntp/htdocs/ST_rpts/T OX72.pdf>. Acesso em: 28 de jul. 2013.

PARDO, P.E; REIS, L.S.L.S. Nutrientes e nutracêuticos em grandes animais. In: ANDRADE, S.F. Manual de terapêutica veterinária. 3. ed. São Paulo: Rocha, 2008. Cap. 29, p.808-814.

PRITI, D.V.; PATEL, J.; VARIA, R.D.; PATEL, J.M.; GHODASARA, B.P.J.; PRAJAPATI, K.S. Effects of sodium dichromate on haematobiochemical parameters in wistar rats. Journal of Pharmacology and Toxicology, v.7, p.58-63, 2012. http://dx.doi.org/10.3923/ipt.2012.58.63

RAZA, M.; AL-SHABANAH, O.A.; EL-HADIYAH, T.M.; AL-MAJED, A.A. Effect of prolonged vigabatrin treatment on hematological and biochemical parameters in plasma, liver and kidney of swiss albino mice, Sci. Pharma., v.70, p.135-146, 2002.

ROBERFROID, M. B. et al. Colonic microflora: nutrition and health. Summary and conclusion of an international life science institute (ILSI) [Europe] workshop held in Barcelona, Spain. Nutr. Rev. V.53, p.127-130, 1995. http://dx.doi.org/10.1111/j.17534887.1995.tb01535.x

SARYAN, L.A.; REEDY, M. Chromium determinations in a case of chromic acid ingestion. Journal Anal Toxicology. v.12, p.162164, 1988.

http://dx.doi.org/10.1093/jat/12.3.162

SEDMAN, R.M.; BEAUMMONT, J.; MCDONALD, T.A.; KROWECH, G.; HOWD, R. Review of evidence regarding the carcinogenicity of hexavalente chromium in drinking water. Journal of Environmental Science and Health, Part C: Environmental Carcinogenesis and Ecotoxicology., v.24, p 155-182, 2006. http://dx.doi.org/10.1080/10590500600614337

SINGH, A.K.; GUPTA, V.K; GUPTA, B. Chromium(III) selective membrane sensors based on Schiff bases as chelating ionophores. Anal. Chim. Acta, v.585, n.1, p.171-8, 2007. http://dx.doi.org/10.1016/j.aca.2006.11.074

SORIANO, G. Probióticos, prebióticos y simbióticos en las enfermedades hepáticas. Gastroenterología y hepatología, v.32, n.1, p.40, 2009.

SOUDANI N.; SEFI, M.; BEN AMARA, I.; BOUDAWARA, T.; ZEGHAL, N. Protective effects of selenium (Se) on chromium (VI) induced nephrotoxicity in adult rats. Ecotoxicol. Environ. Saf., v.73(4), p.671-678, 2010. http://dx.doi.org/10.1016/j.ecoenv.2009.10.002

THE INTERNATIONAL PROGRAMME ON CHEMICAL SAFETY. Environmental Health Criteria, 1 Chromium. Geneva: WHO, 1976. Disponível em: <http://www.inchem. 
org/documents/ehc/ehc/ehc001.htm>. Acesso em: 15 nov. 2013.

TYL, R.W.; MARR, M.; MEYERS, C.B. Developmental toxicity evaluation of chromic acid administered by gavage to CD-1 mice. Research Triangle Institute, Research Triangle Park, Study No. 60C-4808-10/20. November 12, 1991.

U.S. EPA (Environmental Protection Agency) National primary drinking water regulations 40CFR14162 . 2003. [[accessed 3 April 2014]]. Available:

http://edocket.access.gpo.gov/cfr_2003/julqt r/40cfr141.62.htm

WITT, K. L. et al. Nephroprotective effects of methanolic extract of peucedanum grande against acute renal failure induced by potassium dichromate in rats. Toxicologic Pathology, v.41, p.326-342, 2013. http://dx.doi.org/10.1177/0192623312469856

XU, R.Y.; WAN, Y.P.; FANG, Q.Y.; LU, W.; CAI, W. Supplementation with probiotics modifies gut flora and attenuates liver fat accumulation in rat nonalcoholic fatty liver disease model. Journal of Clinical Biochemistry and Nutrition, v.50, n.1, p.72, 2012. http://dx.doi.org/10.3164/icbn.11-38

ZHANG, J.D.; LI, X.L. Chromium pollution of soil and water in Jinzhou. Chinese J. Prev. Med. v.21, p.262-264, 1987. 